\title{
92. Jahresversammlung der DGHNO-KHC 2021 erstmals im Online-Format - eine kritische Bewertung auf Basis der Teilnehmerevaluation
}

\section{First time online $92^{\text {nd }}$ annual meeting of the DGHNO-KHC 2021 - a critical assessment based on the participant's evaluation}

Autoren

Andreas Dietz' ${ }^{1}$, H. J. Welkoborsky², O. Guntinas-Lichius ${ }^{3}$, T. Stöver ${ }^{4}$, M. Wiederkranz ${ }^{5}$, M. Thorleuchter ${ }^{6}$, O. Rosenkranz ${ }^{7}$, T. Deitmer ${ }^{6}$, S. Plontke ${ }^{8}$

Institute

1 HNO-Universitätsklinik Leipzig, Leipzig

2 HNO-Klinik, KRH Klinikum Nordstadt, Hannover, Hannover

3 HNO-Universitätsklinik Jena, Jena

4 HNO-Universitätsklinik Frankfurt a. Main, Frankfurt am Main

5 COCS GmbH, München

6 DGHNO-KHC Geschäftsstelle, Bonn

7 ESTENSIS GmbH, Velten

8 Klinik für HNO-Heilkunde, Kopf- und Halschirurgie Halle, Halle (Saale)

Schlüsselwörter

Online-Kongress, virtuelles Kongressformat, virtuelle Industrieausstellung, Evaluation, Veranstaltungsmanagement

Key words

online meeting, virtual congress format, virtual industrial exhibition, evaluation, event management

online publiziert 24.08 .2021

Bibliografie

Laryngo-Rhino-Otol 2021; 100: 781-790

DOI 10.1055/a-1579-8096

ISSN 0935-8943

(C) 2021. Thieme. All rights reserved.

Georg Thieme Verlag KG, Rüdigerstraße 14,

70469 Stuttgart, Germany

Korrespondenzadresse

Prof. Dr. Andreas Dietz

HNO-Universitätsklinik Leipzig, Liebigstraße 10-14, 04103 Leipzig, Deutschland

Tel.: $+49 / 341 / 9721700$

Fax: $+49 / 341 / 9721709$

andreas.dietz@medizin.uni-leipzig.de, http://www.unileipzig.de/ hno/

\section{ZUSAMMENFASSUNG}

Die 92. Jahresversammlung der DGHNO-KHC wurde im Mai 2021 erstmals aufgrund der Corona-bedingten neuen Rahmenbedingungen komplett in einem Online-Format durchgeführt. Den Teilnehmern aus den Reihen der Mitglieder, Gäste und Industriepartner wurde im Anschluss ein Evaluationsfragebogen der zuständigen Landesärztekammer Nordrhein zugesandt, der inhaltlich um Fragen zu dem neuen virtuellen Format ergänzt wurde. Die Publikation fasst die Auswertung der 187 ärztlichen Rückläufer (10\% der Gesamtteilnehmerzahl) und der 25 Industrieaussteller (60\% der Industrieaussteller) zusammen und stellt ein erstes Fazit auf. In der groben Betrachtung wurde der Kongress von den ärztlichen und wissenschaftlichen Teilnehmern trotz komplett fehlender sozialer und sehr eingeschränkter fachlicher Interaktion überwiegend positiv bewertet. Auf Seiten der Industrie bot sich ein gegenteiliges Bild. Auf die Frage an die ärztlich/wissenschaftlichen Teilnehmenden nach dem zukünftigen Format der nächsten HNO-Jahreskongresse sprachen sich $16 \%$ für einen reinen Online-Kongress, 67 \% für einen Präsenzkongress mit OnlineElementen und lediglich $17 \%$ für einen reinen Präsenzkongress aus. Auf die Frage an die Industrie, welche Art der Ausstellung in Zukunft bevorzugt würde, sprachen sich $68 \%$ für die reine Präsenzausstellung im Rahmen eines Präsenzkongresses aus. 32 \% konnten sich für Präsenz mit Online-Elementen erwärmen. Eine zukünftig komplette Online-Industrieausstellung wurde mit $0 \%$ Zustimmung abgewählt.

\section{ABSTRACT}

First time ever, the $92^{\text {nd }}$ annual meeting of the DGHNO-KHC was carried out completely in an online format in May 2021 due to the new framework conditions caused by the corona pandemic. The participants from the ranks of members, guests and industrial partners were asked to complete an evaluation questionnaire from the responsible State Medical Association of North Rhine, which was supplemented with questions about the new virtual format. The publication summarizes the evaluation of the 187 (10\% of the total number of participants) medical/scientific and 25 industrial exhibitors (60\% of the participating industrial partners) responses and provides an initial conclusion. Roughly speaking, the congress 
was rated positively by the medical/scientific participants despite the total lack of social interaction and scientific open debates. On the industrial side, the picture was different. The medical participants were asked about the future format of the next annual ENT congresses. $16 \%$ were in favor of a purely online congress, $67 \%$ in favor of a face-to-face congress with online elements, and only $17 \%$ in favor of a purely face-to- face congress. The question, which type of exhibition would be preferred in the future for industrial partners, $68 \%$ were in favor of a purely face-to-face exhibition as part of a face-toface congress. $32 \%$ accepted a combination of presence with online elements. A future complete online industrial exhibition was voted out with $0 \%$ approval.

\section{Einleitung}

Nachdem die 91. Jahresversammlung der Deutschen Gesellschaft für Hals-Nasen-Ohren-Heilkunde - Kopf- und Halschirurgie (DGHNO-KHC) 2020 aufgrund der COVID-19-Pandemie kurzfristig abgesagt werden musste und eine im November als komprimierte „Notveranstaltung“ „Essenz 91. Jahresversammlung“, die ursprünglich als Hybridveranstaltung im Paulinum Leipzig geplant war, dann aber Pandemie-bedingt in ein Online-Format umgestaltet werden musste, sahen wir uns in der Phase der massiven zweiten Welle der Pandemie gezwungen, über ein geeignetes und vor allem sicher durchführbares Format für die 92. Jahresversammlung zum 100-jährigen Bestehen unserer Fachgesellschaft nachzudenken. Aufgrund der guten Erfahrungen der Essenz-Veranstaltung im November 2020, durchgeführt von unserer Gesellschaft in enger Zusammenarbeit mit der seit vielen Jahren für uns tätigen Firma ESTENSIS, die zumindest die Durchführung der Mitgliederversammlung 2020, die Ehrungen der wissenschaftlichen Preisträger und die würdige Präsentation der Referate auf ZOOM $^{\circledR}$-Basis ermöglichte, hatten wir uns im Präsidium aufgrund des Corona-Geschehens auf das Abenteuer verständigt, 2021 komplett online zu planen. Begleitet wurde die Entscheidung von einem erheblichen wirtschaftlichen Schaden für unsere Gesellschaft durch die Absage im Jahr 2020, der sich aus den fehlenden Kongresseinnahmen und den vertraglichen Verpflichtungen gegenüber dem Veranstaltungsort ESTREL-Hotel und -Kongresszentrum ergab. Zwar konnte der Schaden aufgrund der guten wirtschaftlichen Verfassung unserer Gesellschaft und der finanziellen Unterstützung vieler Mitglieder (Sonderabgabe) kompensiert werden, doch stand unstrittig im Raum, dass weitere Havarien dieser Dimension unbedingt vermieden werden mussten. Uns war aber auch unstrittig bewusst, dass die Jahresversammlung als wesentliches Jahresereignis unserer Fachgesellschaft kein zweites Mal abgesagt werden darf. Für 2021 stand also fest, dass wir nur in einem Online-Format die gebotene Planungssicherheit erreichen und auf jeden Fall die 92. Jahresversammlung durchführen können ( $\triangleright$ Abb. 1).

Die Herausforderung bzw. Ungewissheit ob des Erfolgs war groß, da es sich um ein komplett neues Format in großem Rahmen, unbekanntes technisches Terrain, die große Bedeutung der 100. Jahresfeier, die Integration von Teilen des 2020-Kongresses und Planung mit einmalig 2 Kongresspräsidenten handelte. Es handelte sich um eine aus dem Stand $100 \%$-Transformation in einen komplett digitalen HNO-Kongress. In einer sehr konzentrierten und einvernehmlichen Arbeitsteilung des gesamten Teams unserer Geschäftsstelle (allen voran Ulrike Fischer, Monika
Thorleuchter, Sonja Hardt), des Generalsekretärs Thomas Deitmer, der Firma COCS (Martina Wiederkranz, Annette Steidle, Claudia Schäfer) und der Firma ESTENSIS (Oliver Rosenkranz) wurde in kurzer Zeit eine weitere Firma (VRtual X) zur Erstellung der Kongressplattform (Streaming-Portal) ausgewählt und beauftragt. Man muss sich vor Augen halten, dass die Entwicklung für Streaming-Plattformen für komplette Online-Kongresse mit mehreren 1000 Teilnehmern erst durch die Pandemie weltweit angekurbelt wurde, und wir uns inmitten einer steilen Lernkurve für Anbieter, veranstaltende Fachgesellschaften und Teilnehmer befanden. Im Rahmen der Vorbereitungen wurde schnell klar, dass unsere Ideen und Anforderungen auch für VRtual X zu kontinuierlichen Neuprogrammierungen und Erweiterungen ihres Produktes geführt haben. Als weiterer technischer Partner ist die Firma „Smart Abstract GmbH“ zu erwähnen, die schon länger mit dem Abstract-Management und der APP betraut ist und bei dem jetzigen Kongress auch an der Umsetzung der Plattform mitgearbeitet hat und mit dem Einbinden des wissenschaftlichen Programms im „Foyer Wissenschaft“ sowie bei „on demand“ mit Einstellen der aufgezeichneten Beiträge betraut wurde. Schließlich hat sich der Thieme-Verlag als Partner für die Kongresspublikationen beteiligt.

Das traditionelle Programm musste aus technischen Gründen in enger Zusammenarbeit mit Ulrike Fischer, Monika Thorleuchter, Susanne Wiegand, Torsten Rahne, Stefan Plontke und Andreas Dietz komplett umgestellt und in 3 parallelen Linien (also nicht 9, wie in einem Kongresszentrum bisher möglich) komprimiert werden. Die Poster, Videos und freien Vorträge wurden frei verfügbar, allerdings ohne Live-Moderationsmöglichkeit in einem „dritten Saal“, der Mediathek, zusammengestellt. Die Präsentationssitzungen unserer Arbeitsgemeinschaften wurden auf einen Tag konzentriert. Der Pflegetag wurde separat auf ZOOM ${ }^{\circledR}$ komplett virtuell abgebildet. Zeitlich ausgelagert und zu späteren Zeitpunkten terminiert wurden die Kurse der HNO-Akademie, die Sitzungen der Chefärzte und Lehrstuhlinhaber, die Geschäftssitzungen der Arbeitsgemeinschaften sowie die Leitliniensitzung. Die Planung wurde über Monate innerhalb wöchentlicher ZOOMMeetings im oben skizzierten Personenkreis sehr produktiv vorangetrieben.

Ein in Präsenz geplanter „Glanztag“ im ESTREL (Mittwoch), an dem die Präsentation der Referate, die feierliche Kongresseröffnung mit Ehrungen, die beiden Wullstein-Lectures aus 2020/ 2021 und die Mitgliederversammlung vorgesehen waren, musste schließlich Corona-bedingt auch in ein komplettes Online-Format umgewandelt werden (inkl. Online-Voting durch einen separaten Anbieter im Rahmen der Mitgliederversammlung der DGHNOKHC und HNO-Akademie). 

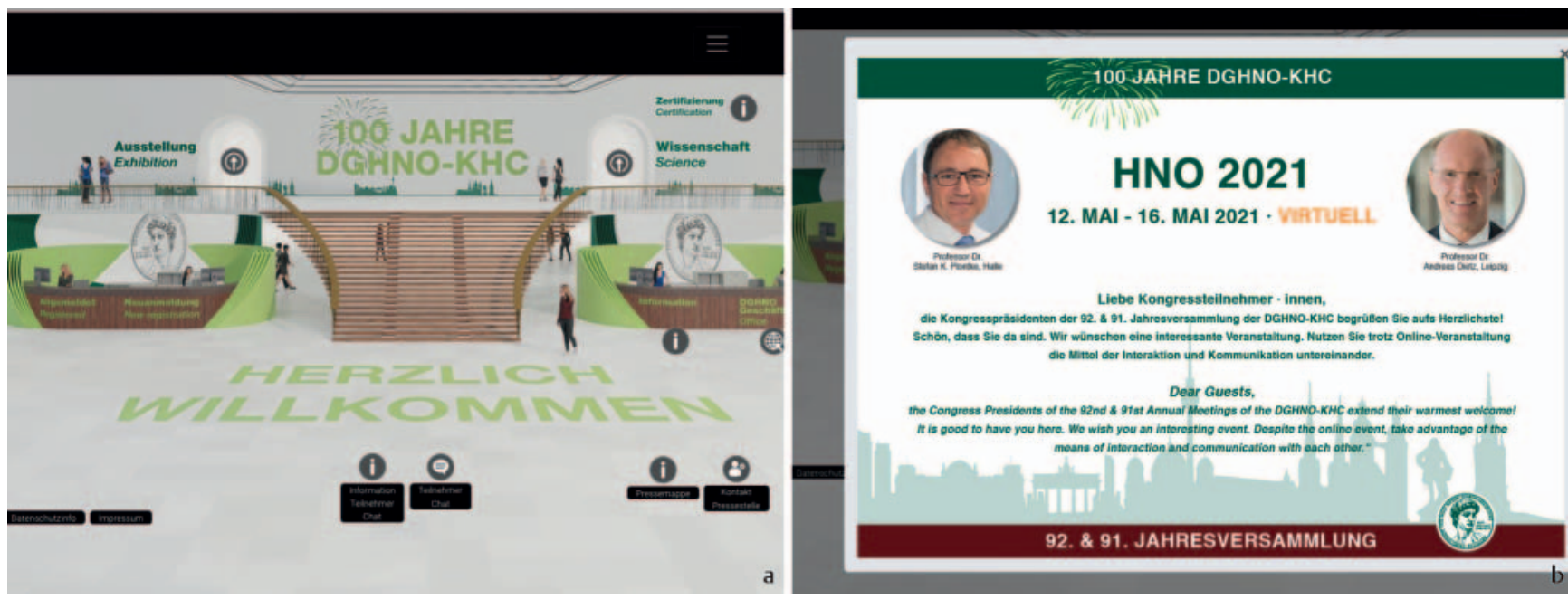

- Abb. 1 a, b Eingangsportal zur 92. Jahresversammlung der DGHNO-KHC.

Die Mehrzahl der Beiträge wurde in Prerecordings voraufgezeichnet und dann auf die Plattform gestellt. Hierzu mussten im Vorfeld verbindliche Termine mit Referenten und Moderatoren mit hohem Planungsaufwand für Veranstalter und Teilnehmer gleichermaßen vereinbart werden. Dies bedeutete für uns alle die Herausforderung der Verfügbarkeit stabiler Internetverbindungen (kabelgebunden besser als WLAN), der Ausstattung mit tauglichen Endgeräten, der Kamera- und Tonqualität (Head Sets, Position der Kamera), der geeigneten Hintergründe, des Umgangs mit ZOOM, des strikten Zeitmanagements etc. ... Die meisten Diskussionen der Rundtische und Boards wurden live übertragen. Als Besonderheit wurde ein täglicher „President's Corner“ live ab 19:00 Uhr eingeführt, der den Teilnehmern einen kommentierten Tagesausklang mit dem Präsidenten und Ehrenpräsidenten (Hans Peter Zenner, Heinz Maier) ermöglichte ( $\bullet$ Abb. 2). Zu den live stattfindenden Kongressveranstaltungen gab es einen Teilnehmer-Chat, der Fragen zur Diskussion aus dem Auditorium zuließ (SLIDO, Schulung der Moderatoren im Vorfeld). Leider war dies bei den Postern, freien Vorträgen und Videos technisch auf der VRtual X-Kongress-Plattform „noch“ nicht möglich. Auch konnten wir technisch keine verlässliche Interaktionsmöglichkeit zwischen den Teilnehmern herstellen (Chat-Funktion, bildliche Face-to-Face-Verbindung), die sicher ein verbessertes Kommunikationserlebnis erzeugt hätte.

Für die Industrie wurde auf der Plattform eine virtuelle Ausstellung angeboten, die eine Bewegung der Teilnehmer in 3 Hallen mit virtuellen Ständen und verschiedenen Kontaktmöglichkeiten mit Vertretern der Industrie erlaubte ( $\triangleright$ Abb. 3). An den Ständen konnten Links zu Videopräsentationen und Live-Chats mit Vertretern angewählt werden. Zusätzlich wurden Industriesymposien angeboten, die im Programm zu festen Zeiten verortet wurden. Insgesamt haben 39 Firmen Stände gebucht, 14 Firmen Industriesymposien (davon 3 Premium-Symposien) und eine Firma hat sich lediglich mit Logo auf der Kongress-Webseite beteiligt (insgesamt also 42 kongressaktive Industriepartner).

Insgesamt haben sich über die 4 Tage des Kongresses 1801 Teilnehmer zu unterschiedlichen Zeiten eingewählt ( $>$ Tab. 1).

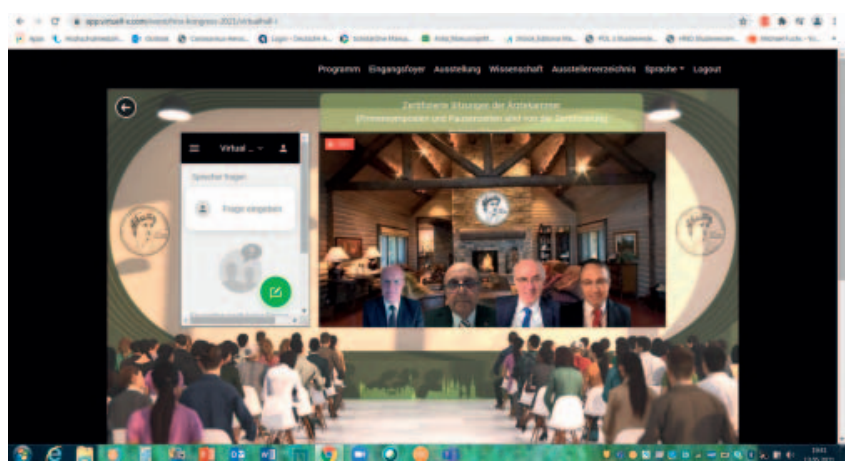

Abb. 2 President's Corner.

- Tab. 1 Auflistung der Teilnehmenden- und Industriebeteiligung.

Teilnehmende insgesamt: 1801,

davon:

- 1045 Teilnehmer Kongress

- 223 Teilnehmer Akademie

- 44 nur Mitgliederversammlungen

- 179 personalisierte Teilnehmer aus der Industrie

- 33 Teilnehmer Presse

- 34 HNO-Pflegetag

- 134 Gast-/Kongress-Freikarten

- 109 Medizinstudenten

Industriepartner/Firmen: $\mathbf{4 2}$

- 39 Aussteller auf virtueller Industrieausstellung

- 11 Symposien + 3 Premium-Symposien (2 ausschließlich Symposium)

- 1 ausschließlich Beteiligung mit Logo auf Kongress-Webseite

Lediglich 1-mal kam es am ersten Tag zu einem überlastungsbedingten Zentralserverabsturz, der eine ca. 10-minütige Verzögerung (inkl. Schockmoment für das gesamte Organisationsteam, frei nach Apollo 13 „Houston, wir haben ein Problem!“) der Online-Verbindung bewirkte. Ansonsten lief die Veranstaltung 

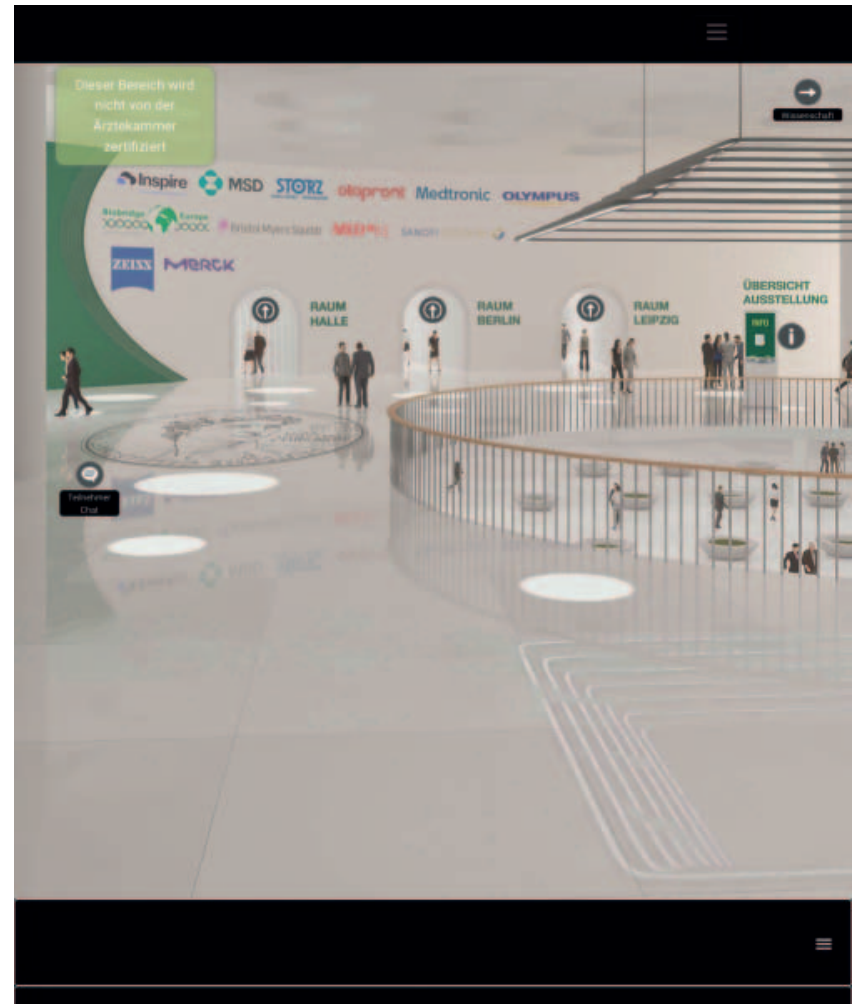

Zertifizierung

\section{(1) Ausstellungshalle 1}

\section{(†) Ausstellungshalle 2}

\section{(A) Ausstellungshalle 3}

Abb. 3 Portal zur Industrieausstellung.

reibungslos nach Plan. Mit > 600 Teilnehmenden waren die Referate die am besten besuchten Symposien. Die Industriesymposien zogen jeweils zwischen 224 und 497 Teilnehmende an. Die übrigen Rundtische/Symposien zogen zwischen 141 und 517 Interessierte an. Bemerkenswert erschien, dass der Diskussion zur Frauenquote 400 Eingewählte beiwohnten. Auch die Sitzungen der Arbeitsgemeinschaften waren selbst in den frühen Stunden des Sonntags (z. B. für die Arbeitsgemeinschaft Klinische Immunologie, Allergologie und Umweltmedizin mit 403 Teilnehmern) sehr gut besucht. Etwas weniger frequentiert war die Mediathek (freie Vorträge, Poster, Videos) mit 50-190 Einwahlen. Der Zugang des Kongressportals mit sämtlichen Beiträgen wurde für 2 Monate nach dem Kongress für alle registrierten Teilnehmer aufrechterhalten und dann aus Kostengründen (Server-Speicherkapazität) geschlossen.

Im Anschluss der Veranstaltung wurde an alle Teilnehmer sowie unsere Partner aus der Industrie ein Evaluationsbogen geschickt, dessen Auswertung in der folgenden Publikation dargestellt werden soll. Neben dem Rücklauf der Evaluation fließen auch teilweise ausführliche Telefonate und persönliche Rückmeldungen aus Industrie und Mitgliederbereich ein. Die Publikation soll einen Beitrag für die Weiterentwicklung möglicher digitaler Elemente für unser zukünftiges Jahreskongressformat nach der Pandemie leisten.

\section{Ergebnisse der Evaluation}

Die folgende Darstellung der Evaluation wird in 2 Teile untergliedert, die sich mit dem Rücklauf der Mitglieder und registrierten Gäste der DGHNO-KHC und mit dem Feedback der Industrie befassen. Für die Evaluation wurde der von uns nach neuen virtuellen Kriterien ergänzte Fragebogen „Fortbildungsevaluation für anerkannte Veranstaltungen“ der für den Kongress zuständigen Landesärztekammer Nordrhein eingesetzt. Der Fragebogen wurde webbasiert über „SurveyMonkey ${ }^{\circledR “}$ adressiert und beantwortet.

\section{Rücklauf aus den Reihen der Mitglieder und Gäste}

Von den insgesamt 1801 registrierten Teilnehmern haben 187 die Beantwortung vollständig abgeschlossen (82\% Fachärzte, $17 \%$ Assistenten in Weiterbildung, $1 \%$ nichtärztliche Teilnehmer). $73 \%$ haben den Kongress nach dem Schulnotensystem mit gut (46\%) bzw. sehr gut (27\%) bewertet. Als mangelhaft bzw. ungenügend haben $3 \%$ den Kongress bewertet. Auf die Frage nach dem zukünftigen Format der nächsten HNO-Jahreskongresse sprachen sich $16 \%$ für einen reinen Online-Kongress, $67 \%$ für einen Präsenzkongress mit Online-Elementen und lediglich $17 \%$ für einen reinen Präsenzkongress aus ( $\triangleright$ Abb. 4).

Bezüglich der inhaltlichen Elemente des Kongresses verteilten $83 \%$ die Noten gut und sehr gut ( $\triangleright$ Abb. $\mathbf{5}$ ).

Bei dem Kongress wurden die verschiedenen Angebote in unterschiedlicher Frequenz genutzt. $93 \%$ gaben an, aufgezeichnete Vorträge mit Live-Präsenz der Vortragenden genutzt zu haben. 33 \% besuchten lediglich die freien Vorträge und 83 \% nutzten insgesamt die Mediathek mit freien Vorträgen und E-Postern. $43 \%$ gaben an, die Angebote der Mediathek nur während der Kongresstage, 53 \% sowohl während als auch nach dem Kongress und $4 \%$ ausschließlich nach dem Kongress genutzt zu haben. $62 \%$ gaben an, in zukünftigen Kongressen das alleinige Angebot der Poster über ein Internetportal mit nachhaltiger Verfügbarkeit über 2 Monate vorzuziehen. Dies wurde von $38 \%$ verneint.

Das Online-Format wurde nach unterschiedlichen Gesichtspunkten bewertet. $70 \%$ gaben als positives Argument an, dass es die Möglichkeit eröffnete, die virtuellen (parallelen) Veranstaltungen zu hören (was bei reiner Präsenz nicht möglich ist). $65 \%$ sahen einen Vorteil in dem Umstand, nicht reisen zu müssen. $43 \%$ sahen einen Nachteil in der fehlenden persönlichen Diskussion. $71 \%$ beklagten den Nachteil, der dem fehlenden persönlichen Kontakt und Austausch innewohnte.

$63 \%$ haben die virtuelle Industrieausstellung besucht. $37 \%$ gaben an, die Industrieausstellung nicht besucht zu haben. In - Abb. 6 ist das Meinungsbild zur virtuellen Industrieausstellung zusammengetragen. 31 \% fanden die Darbietung informativ, $28 \%$ gut strukturiert. Lediglich $19 \%$ gaben an, dass sie neue Informationen über Medizintechnik erhalten hätten. Allerdings gaben $66 \%$ an, dass ihnen der persönliche Kontakt zu den Vertretern der einzelnen Firmen fehlte ( $>$ Abb. 6). $58 \%$ gaben an, dass sie bei zukünftigen Kongressen das zusätzliche Angebot von mehr 
F4: Was würden Sie künftig eher begrüßen, sofern möglich? (nur 1 Antwort möglich)

Beantwortet: 187 Obersprungen: 0

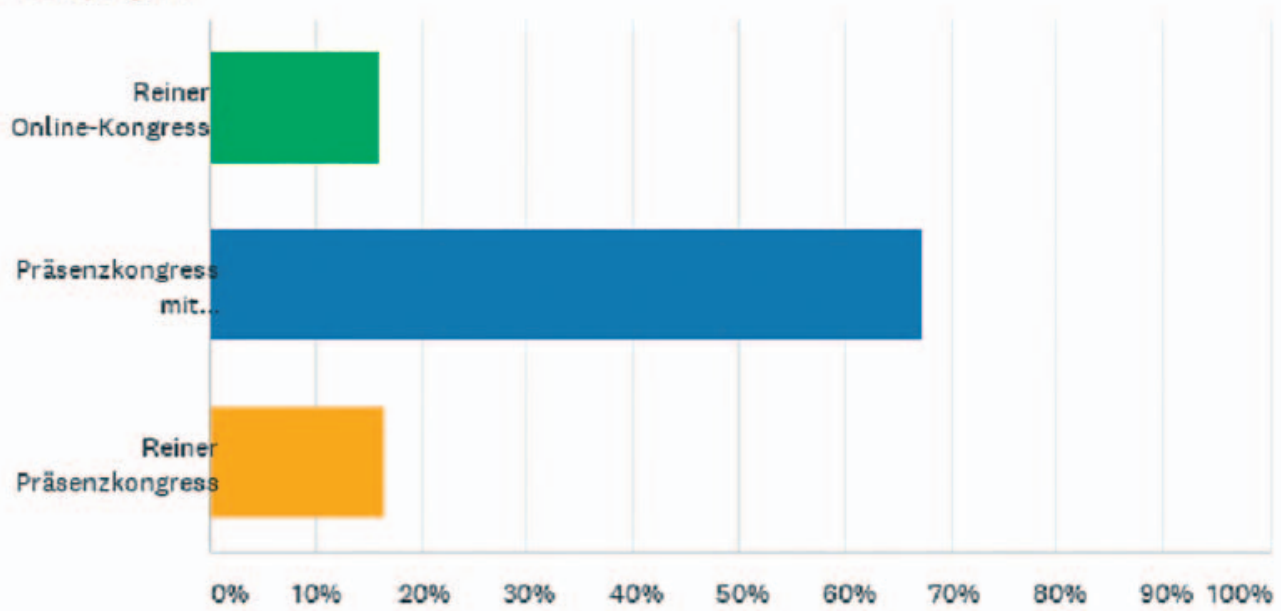

Powered by the SurveyMonkey

- Abb.4 Bevorzugtes zukünftiges Kongressformat.

F5: Wie beurteilen Sie die inhaltlichen Elemente des Kongresses? (nach Schulnoten 1-6)

Beantwortet: 187 Obersprungen: 0

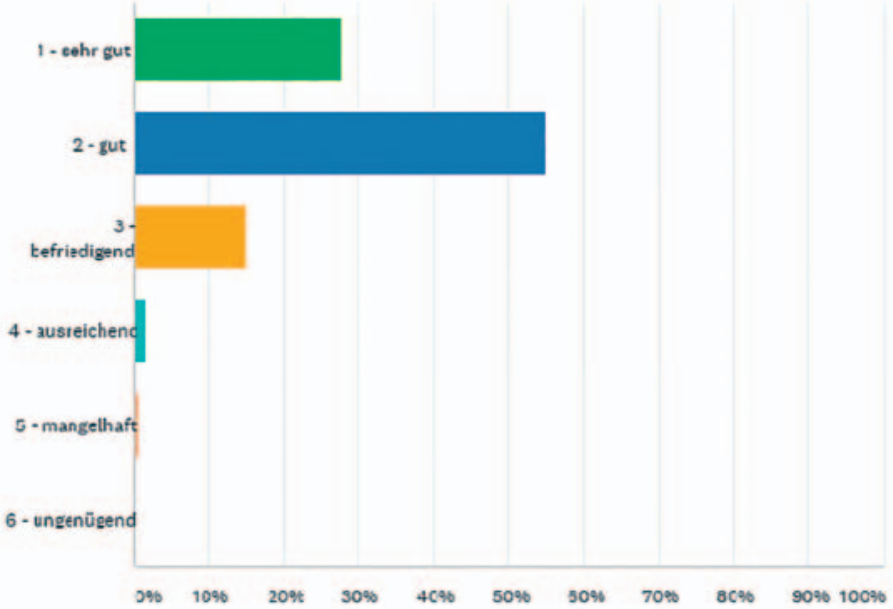

Powered by the SurveyMonkey

- Abb. 5 Inhaltliche Bewertung des Kongresses. 


\section{F12: Die virtuelle Industrieausstellung fand ich: (mehrere Antworten möglich)}

Beantwortet: 163 Obersprungen: 24

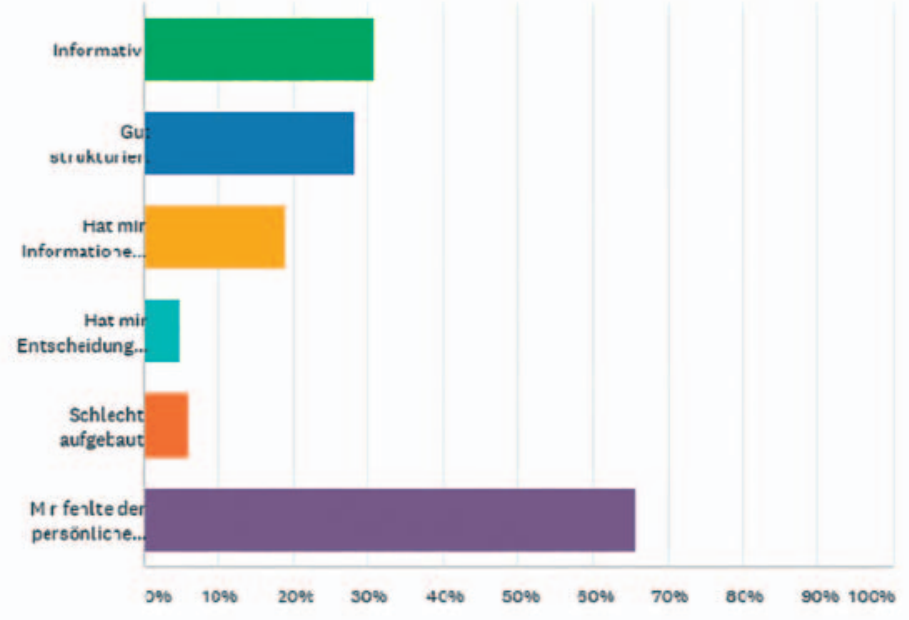

Powered by ath SurveyMonkey

- Abb. 6 Meinungen zur virtuellen Industrieausstellung.

virtuellen Bestandteilen der Industrieausstellung nicht nutzen würden. Im Freitext wurde angegeben, dass aufgrund des sehr dichten Programms nur wenig Zeit verblieben sei, die Industrieausstellung zu besuchen.

$74 \%$ der Teilnehmer fanden, dass man bei zukünftigen Kongressen Teile des Programms (z. B. Geschäftssitzungen der AGs, einige Industriesymposien etc.) online nach Abschluss der reinen Kongresstage anbieten solle.

Geäußerte Kritik im Freitext:

Bezüglich der Zertifizierung durch die Landesärztekammer Nordrhein gab es aufgrund des neuen Formats im Vorfeld erheblichen Abstimmungsbedarf. Vonseiten der Teilnehmer gab es Kritik, dass nicht klar gewesen wäre, dass Fortbildungspunkte nur bei komplett absolvierter Online-Veranstaltung anerkannt wurden und bei manchen die Punktevergabe aus technischen Gründen inkorrekt gelaufen sei. Des Weiteren wurde kritisiert, dass die ePoster teilweise unleserlich, umständlich zu vergrößern und schwer zu finden (fehlende Suchfunktion) waren und nicht diskutiert wurden. Zu der Internet-Plattform gab es kritische Stimmen, die sich auf die fehlende Rückmeldung der eingewählten Teilnehmer bei Postern und freien Vorträgen in der Mediathek, die insgesamt wahrgenommene Schwerfälligkeit und die teilweise schlechte akustische Übertragung mangels Headsets bzw. suboptimaler Endgeräte der Vortragenden bezogen. Ein Vorschlag brachte wegen des HNO-spezifischen relevanten Anteils möglicher hörgeschädigter Teilnehmer auch den notwendigen Einsatz eines Schriftdolmetschers (Untertitel) in die Diskussion. Mehrere Kritiker mahnten die fehlende Interaktion zwischen den Teilnehmern an.
Geäußertes Lob im Freitext:

Neben zahlreichem Lob an Format und Organisation fasste eine Stimme die Äußerungen mehrerer Teilnehmer gut zusammen: „Am schönsten wäre ein Kongress, der live und virtuell ist. Wobei die Verfügbarkeit in beiden Formen für alle Angebote sein sollte und danach on demand. Bisher musste man sich beim Live-Kongress immer einen Vortrag aussuchen, und wenn mehrere interessante gleichzeitig waren, hat man die anderen für immer verpasst.“ Eine weitere Stimme äußerte: „Ein echter Vorteil für Klinikärzte mit regelmäßigen Diensten ist, dass man Online-Kongresse an den dienstfreien Tagen besuchen kann, auch wenn man zwischendrin Dienst hat. Das ist bei Präsenzkongressen mit Anreise nicht möglich. Hierfür wäre es schön, wenn man die Online-Vorträge tageweise buchen könnte." Auch die Akademie wurde überwiegend gut angenommen und die zeitliche Verschiebung in die Folgewoche sehr positiv bewertet. Das „on demand“Prinzip wurde überwiegend gelobt, allerdings der Wunsch geäußert, die Beiträge länger als 2 Monate verfügbar im Netz zu belassen. Auch der „President's Corner“ wurde positiv gewürdigt. Die fehlende soziale Interaktion wurde mit Blick auf die gegebenen Umstände kommentiert: „Es gab wenig Ablenkung: Das war vorteilhaft, da ich deutlich mehr die Informationen nun in meinen Klinikalltag einfließen lassen kann, und es war auch etwas nachteilig, da es keinen Gesellschaftsabend mit Kollegen gab. Ein Thema lautete u. a. Frauenquote: Durch die Online-Variante waren Familie, Beruf und Kongress deutlich besser im Einklang zu bringen...; der Kongress war unter den obwaltenden Umständen vermutlich das Beste, was zu realisieren war. Das der Pandemie geschuldete Format ersetzt aber nicht das Persönliche eines konventionellen 


\section{F1: Wie bewerten Sie die virtuelle Industrieausstellung insgesamt:(Schulnoten 1-6)}

Beantwortet: 25 Obersprungen: 0

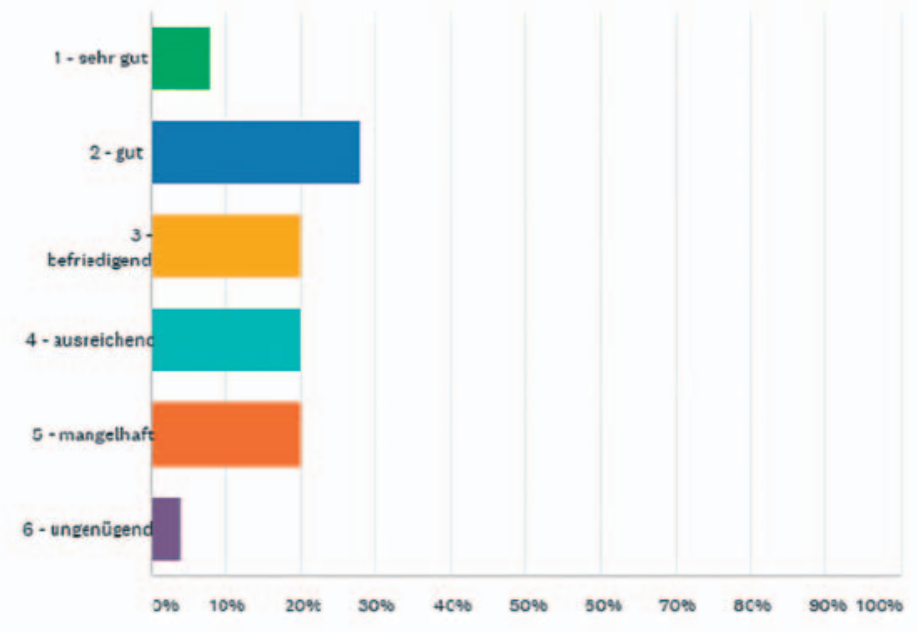

Powered by the SurveyMonkey

- Abb. 7 Bewertung der virtuellen Industrieausstellung aus Sicht der Industriepartner.

Kongresses. Dies bitte ich aber, nicht als Kritik an den Veranstaltern aufzufassen.“

\section{Rücklauf aus den Reihen der Industrie}

Insgesamt beantworteten 25 Industriepartner (von insgesamt 42 beteiligten) die Evaluationsfragen. Hier ergab sich ein zu den ärztlichen Teilnehmern unterschiedliches Bild. Lediglich $36 \%$ vergaben die Schulnoten gut und sehr gut. Jeweils $20 \%$ bewerteten den Kongress als befriedigend, ausreichend und mangelhaft. $4 \%$ vergaben die Note ungenügend ( $\boldsymbol{A} \mathbf{A b b} \mathbf{~} \mathbf{7})$.

Von den 25 antwortenden Industriepartnern waren 64\% mit einem Stand, $4 \%$ ausschließlich mit einem Industriesymposium, $32 \%$ mit Stand und Symposium und $0 \%$ lediglich mit Sponsoring auf dem Kongress engagiert. $24 \%$ fanden die Ausstellung gut strukturiert. $48 \%$ gaben an, die Ausstellung war leicht zu besuchen. $8 \%$ votierten für „unübersichtlich“ und 20 \% für „unpersönlich“. Geradezu desaströs zeichnete sich das Meinungsbild zur Möglichkeit des persönlichen Austauschs ab. 52 \% vergaben die Note „ungenügend“ ( $\triangleright \mathbf{A b b}$. 8).

$92 \%$ gaben an, insgesamt weniger als 10 Online-Kontakte verbucht zu haben. Lediglich $4 \%$ hatten mehr als 100 Kontakte. $52 \%$ der Industriepartner beschrieben das Preis-Leistungs-Verhältnis der Stände/Symposien als „mangelhaft“ bzw. „ungenügend“ $(\triangleright$ Abb. 9).

$84 \%$ gaben an, dass sie nahezu keine Geschäfte (mangelhaft/ ungenügend) im Rahmen der Ausstellung abschließen konnten. Auf die Frage, welche Art der Industrieausstellung in Zukunft bevorzugt würde, votierten $68 \%$ für die reine Präsenzausstellung im Rahmen eines Präsenzkongresses. 32 \% sprachen sich für Präsenz mit Online-Elementen aus. Eine zukünftige komplette Online-Industrieausstellung wurde einstimmig abgewählt. Erfreulicherweise reagierte niemand mit einer Ansage, zukünftig nicht mehr an einer Ausstellung teilnehmen zu wollen. $80 \%$ fanden die Idee gut, für zukünftige Kongresse die Teilnahme der Firma an einer Kombination aus Beteiligung am Jahreskongress plus überjähriger Beteiligung an Fortbildungsveranstaltungen des HNOeCampus zu ermöglichen.

\section{Geäußerte Kommentare im Freitext (überwiegend Tadel)}

Die im Freitext geäußerten Kommentare waren überwiegend von Kritik und Tadel geprägt. Die Kontaktaufnahme mit den Besuchern war deutlich erschwert, da überwiegend sehr anonymer Austausch. Die Möglichkeit eines interaktiven Chats mit auch bildlicher Kontaktaufnahme und damit Ermöglichung eines direkten persönlichen Dialogs wäre hilfreich gewesen. Es war nicht klar, aus welcher Gruppe (Kollege aus der Industrie oder Arzt, niedergelassen oder Kliniker) die Kontaktaufnahmen kamen. Zwei Kommentare brachten es zusammengefasst gut auf den Punkt: „Anwender sehen keinen Mehrwert an einem virtuellen Stand. Produkte muss man anfassen, Menschen muss man in die Augen schauen können. Keines der Ziele (z. B. Markenaufbau/Festigung von Beziehungen/Gewinnung von Neukunden etc.), die man mit so einer Veranstaltung verfolgt, kann erreicht werden. Aus Sicht der Industrie kann es daher nur Präsenzveranstaltungen geben...; Kongress vor Ort hat nicht nur Ziel des Sales-Leads, sondern auch 


\section{F4: Als Firma konnten wir mit den Besuchern in engen Austausch und} Kontakt treten (Schulnoten: 1 stimmt $100 \%$ ig bis 6 stimmt gar nicht)

Beantwortet: 25 Obersprungen: 0

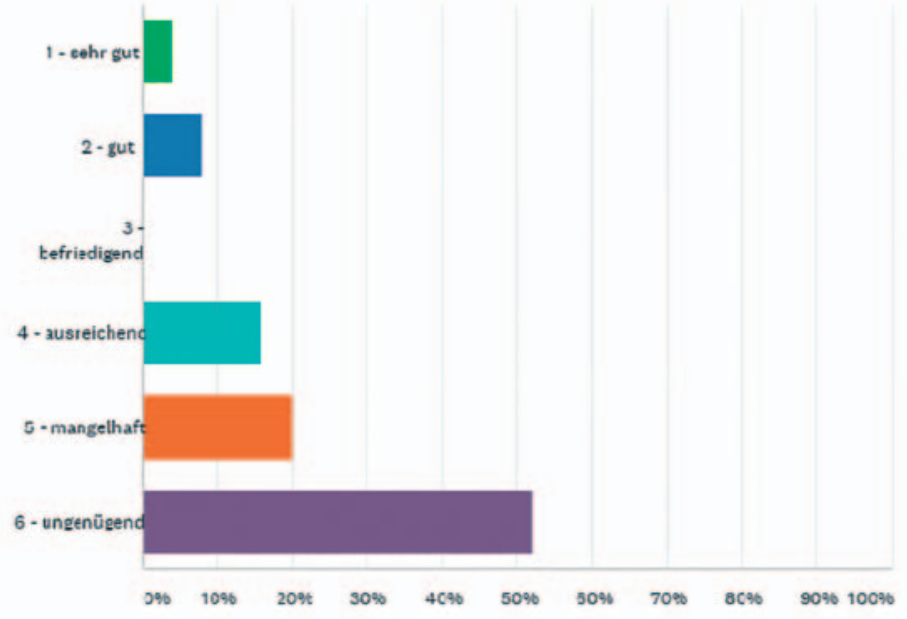

Powered by ath SurveyMonkey

- Abb. 8 Bewertung der Firmen zur Möglichkeit des persönlichen Austauschs mit den Kongressteilnehmern.

F6: Das Preis-Leistungsverhältnis für die Stände/Symposien war (Schulnoten: 1 sehr gut bis 6 völlig inadäquat)

Beantwortet: 25 Obersprungen: 0

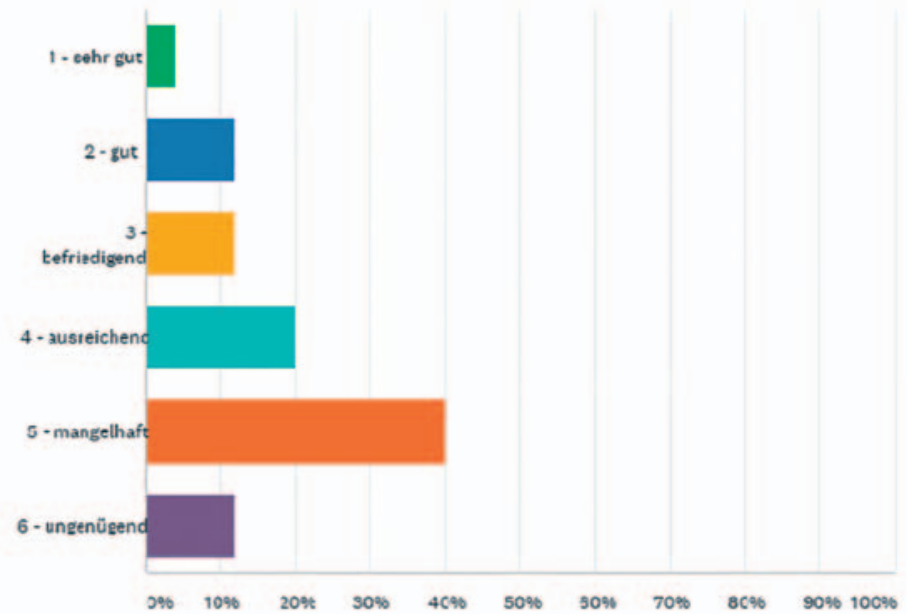

Powered by ath SurveyMonkey

- Abb.9 Bewertung des Preis-Leistungs-Verhältnisses aus Sicht der Industriepartner. 
Kontakte mit Meinungsbildern zu pflegen und neue zu knüpfen. Dieser Aspekt war in der virtuellen Welt natürlich nur begrenzt möglich bzw. wurde nur begrenzt genutzt. Vonseiten der Analytics war der Kongress also leider nicht erfolgreich."

\section{Diskussion und erstes Fazit}

Die Corona-Pandemie hat den Rahmen für die 92. Jahrestagung unserer Fachgesellschaft vorgegeben. Dieser aus den behördlichen Restriktionen definierte Rahmen erlaubte neben dem stattgefundenen Online-Format lediglich die erneute ersatzlose Absage analog zu 2020. Der große motivierende Rücklauf aus den Reihen der Mitglieder ermutigte das Präsidium, den Kongress „trotz“ Online-Format zu organisieren und stattfinden zu lassen. Die beiden Kongresspräsidenten bedanken sich an dieser Stelle ausdrücklich für die wunderbare Zusammenarbeit aller eingangs erwähnten großartigen Personen, die Unglaubliches in kurzer Zeit geleistet und damit einen historisch besonderen Kongress vorbereitet und begleitet haben. Besonders deshalb, da sich einiges (hoffentlich) so schnell nicht wiederholen wird, wie die Corona-Restriktionen und die Fusion zweier komplementärer Jahreskongressprogramme in ein komplett neues virtuelles digitales Format ohne jegliche Vorerfahrung (sozusagen ein großes Experiment mit vielen Unbekannten).

Die technischen Möglichkeiten gab es zwar teilweise bereits vor Corona, doch wurden die Streaming-Plattformen, um solch große Kongresse online anbieten zu können, erst durch die Pandemie ab 2020 als verfügbare Produkte professionalisiert. Etwas weiter waren zu Beginn der Pandemie die Cloud-Dienste für Kleinkonferenzen, wie beispielsweise ZOOM ${ }^{\circledR}$, Skype for Business ${ }^{\circledR}$, Cisco WebEx ${ }^{\circledR}$ Meetings ${ }^{\circledR}$, LogMeln ${ }^{\circledR}$, GoToMeeting ${ }^{\circledR}$, Grandstream IPVideo-Talk Web Meetings ${ }^{\circledR}$, Lifesize Cloud ${ }^{\circledR}$, Microsoft Teams ${ }^{\circledR}$ und Unify Circuit ${ }^{\circledR}$. Diese Cloud-Dienste haben sich überwiegend in der internationalen Geschäftswelt (weniger in der Medizin) zur einfacheren internen und externen Kommunikation entwickelt, bekamen aber im Rahmen der Pandemie einen unglaublichen Entwicklungsschub. Beispielsweise wurde der „President's Corner" in dem Lounge-Format erst durch eine Weiterentwicklung von $\mathrm{ZOOM}^{\circledR}$, die einen Monat vor Kongress auf den Markt kam, möglich (Danke an Oliver Rosenkranz, ESTENSIS). Der technische Aufwand im Hintergrund war immens, wie die - Abb. 10 aus dem Koordinationszentrum der Firma ESTENSIS deutlich macht.

Für die Medizin haben sich Online-Formate als neue Fortbildungs-/Meeting-Tools seit 2020 unter Corona sehr gut entwickelt und werden sicher auch nach Corona bestehen bleiben, da lange Anreisen verbunden mit zeitlich deutlich höherem Aufwand wegfallen. Beispiele sind die mittlerweile etablierten 2-3-StundenFormate wie HNO-eCampus der DGHNO-KHC und die Webinare des BVHNO (bis zu 1374 Teilnehmende BVHNO-Webinar März 2021), die es ermöglichen, bequem nach Dienst/Praxis in vertrauter Umgebung, hochkonzentriert und ablenkungsfrei fachlich neue Inhalte aufzunehmen. Das bestechende Moment, sich in die Veranstaltung pünktlich ohne Vorlauf einwählen zu können und nach Abschalten direkt zu Hause zu sein, ist vor allem für „Prä-Corona“ beruflich Vielreisende, aber auch für Mütter und Vä-

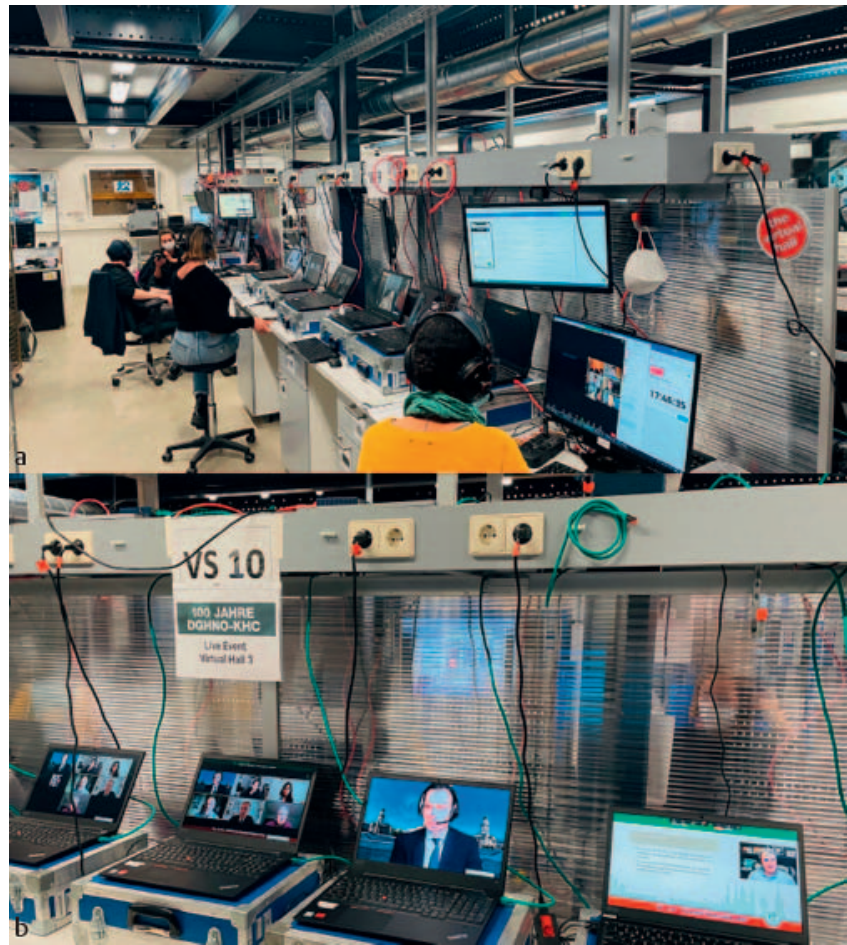

- Abb. 10 a, b Momentaufnahmen aus dem ESTENSIS-Koordinationszentrum während der 92. Jahrestagung der DGHNO-KHC.

ter ein wahrer Segen. Auch längere Seminare, wie beispielsweise die Webinare "Immunmodulation in der Kopf-Hals-Onkologie“ (MedKomAkademie, 2 Tage) oder „Repetitorium HNO - Facharztwissen kompakt“ zusammen mit der Jungen HNO unserer Fachgesellschaft (MedKomAkademie, 4 Tage) mit jeweils deutlich mehr Online-Teilnehmenden als vor Corona, laufen gut und werden in den Evaluationen auch nach Corona als willkommene OnlineFormate gewünscht.

Da der Jahreskongress der DGHNO-KHC mehrere Ziele verfolgt, war bereits im Vorfeld für uns schmerzlich klar, dass eine komplette Digitalisierung bzw. Online-Version mit Stand der Technik 2020 einer Reihe von Zielen nicht gerecht werden kann. Allen voran ist das Ziel des Kennenlernens bzw. Vernetzens der jüngeren und das persönliche Wiedersehen der älteren und bekannteren Mitglieder hervorzuheben: Der Kongress als großes jährliches Familientreffen, mit einer feierlichen Eröffnungsveranstaltung als Gemeinschaftserlebnis mit unverzichtbaren emotionalen Elementen, persönlichen Begegnungen in der Industrieausstellung bzw. am Rande des Kongresses und die wunderbaren Abendveranstaltungen, der Besuch einer schönen Stadt, das Schließen vieler neuer Freundschaften und die herausfordernden ersten freien Vorträge vor Fachpublikum mit anschließender persönlicher Diskussion mit Augenkontakt. Auch wenn durch mittlerweile weit entwickelte Plattformen über erkennbare persönliche Avatars Begegnungen der Teilnehmenden auch auf einem Internetkongress technisch möglich sind (man erkennt das Gegenüber im gleichen virtuellen Raum auf dem Bildschirm und kann durch Anklicken eine Face-to-Face-Verbindung herstellen), wird wahrscheinlich auch die beste virtuelle Illusion die oben 
genannte persönliche „anfassbare“ Begegnungskultur nicht ersetzen können. Auch erscheint unstrittig, dass ein persönliches Kennenlernen auf virtueller Basis nicht die gleiche Nachhaltigkeit und das gewünschte Entstehen einer tiefen Verbundenheit zu der eigenen Fachgesellschaft bewirken wird. Auch wenn wir insgesamt 1801 Teilnehmer online begrüßen durften, liegen unsere bisherigen Kongressteilnahmen deutlich über 2300 mit signifikant höherer Industriebeteiligung.

Die Evaluation macht deutlich, dass vor allem die Online-erprobten ärztlichen Teilnehmenden den Kongress unter dem Aspekt Fortbildung sehr intensiv, aber auch selektiv genutzt haben. Die geringeren Zahlen in der Mediathek und sehr geringen Beteiligungen an der Industrieausstellung machen dies deutlich. So wundert es also nicht, dass gerade die Industriepartner, die genau auf diese persönlichen Netzwerke und Verbindungen setzen, von einer ausschließlichen Online-Kommunikation am wenigsten profitieren. Der Vergleich zu dem Kongress vor Corona spricht für sich: Im Jahr 2019 hatten sich 100 Firmen für den Präsenzkongress mit Ständen auf der Industrieausstellung engagiert, d. h. dass sich im Jahr 202158 Firmen zurückgezogen haben. Größtenteils handelte es sich um Firmen, die im Jahr 2019 eher kleine Standflächen gemietet hatten. Für die Industrie ist also gerade der persönliche Kontakt zu den verschiedenen Arztgruppen (Weiterbildungsassistenten/Nachwuchs, Fachärzte in der Niederlassung, Oberärzte und Chefärzte in Kliniken) extrem wichtig, um die Sichtweise der Meinungsbildner zu kennen und verbindliche Kontakte, die wichtig für das „Branding“ sind, zu erzeugen. Leider ersetzt eine virtuelle Industrieausstellung, auch wenn sie noch so schön gestaltet ist, nicht die realen großen Hallen, in denen sich das Kongresspublikum in dem großen Gewimmel der Ausstellung versammelt. Zum Nachteil für die Industrie wird auch der zeitliche Einsatz der Teilnehmenden, die selektiv auf einige Veranstaltungen fokussierten, um die restliche Zeit womöglich mit Arbeit in Klinik und Praxis bzw. Wirken in der privaten Umgebung verbrin- gen. Wie auch die Evaluation bestätigt (92\% der Firmen gaben an, insgesamt weniger als 10 Online-Kontakte verbucht zu haben), haben sich die Teilnehmenden überwiegend auf das Programm konzentriert und die Industrieausstellung vernachlässigt. Lediglich die im Programm ausgewiesenen Industriesymposien wurden recht gut angenommen und auch von Industrieseite gut bewertet.

Es wird spannend sein, welche virtuellen Kongressanteile sich nach diesem digitalen Experiment der 92. Jahresversammlung in den kommenden Jahresversammlungen etablieren werden. Hierzu hat die Evaluation Impulse gegeben: Die ärztlichen Teilnehmenden wünschen sich nur noch zu $17 \%$ einen reinen Präsenzkongress. 83 \% sprachen sich für die Berücksichtigung virtueller Elemente aus. Das immer wieder geforderte Hybridformat, als Präsenz und parallele Aufzeichnung bzw. interaktive Schaltung für Online-Teilnehmer, wäre natürlich ideal. Aktuell ist eine solche Doppelstruktur noch sehr teuer, da letztlich 2 Kongresse technisch realisiert werden müssten. Zu erwarten ist, dass sich die Kongresstechnik weiterentwickelt und wir auf auch wirtschaftlich interessante Angebote hoffen dürfen.

Durchaus heute schon realisierbar ist die Aufzeichnung von live gehaltenen Vorträgen und Rundtisch-Diskussionen, die den Kongressteilnehmern dann für eine gewisse Zeit zur Nachbereitung online zur Verfügung gestellt werden. Hiermit lässt sich zumindest ein Manko eines Präsenzkongresses, nämlich, dass man nur jeweils eine Parallelveranstaltung besuchen kann und man evtl. auch Interesse an einem gleichzeitig in einem anderen Saal stattfindenden Beitrag hätte, beheben.

Interessenkonflikt

Die Autorinnen/Autoren geben an, dass kein Interessenkonflikt besteht. 